Reporte Interno \# 166

Departamento de Matemáticas

CINVESTAV del IPN

Mexico City, 1994
To appear in

Mathematical Methods in

Applied Sciences

\title{
The Riesz-Clifford Functional Calculus for Non-Commuting Operators and Quantum Field Theory*
}

\author{
Vladimir V. Kisil ${ }^{\dagger}$ \\ e-mail:vkisil@mvax1.red.cinvestav.mx \\ Enrique Ramírez de Arellano \\ e-mail:eramirez@mvax1.red.cinvestav.mx \\ Departamento de Matemáticas, CINVESTAV del I.P.N., \\ Apartado Postal 14-740, 07000, México, D.F. México \\ fax: (525)-752-64-12
}

November 24, 1994

\begin{abstract}
We present a Riesz-like hyperholomorphic functional calculus for a set of non-commuting operators based on the Clifford analysis. Applications to the quantum field theory are described.
\end{abstract}

*This work was partially supported by CONACYT Project 1821-E9211, Mexico.

$\dagger$ On leave from the Odessa State University.

Keywords and phrases. Functional calculus, Weyl calculus, Riesz calculus, Clifford analysis, quantization, quantum field theory.

1991 Mathematical Subject Classification. Primary: 47A60; Secondary: 81T10. 


\section{Contents}

$\begin{array}{lll}1 & \text { Introduction } & 2\end{array}$

2 Different Approaches to Functional Calculus 4

2.1 Two Procedures of Functional Calculus . . . . . . . . . . . . . 4

2.2 Functional calculus: The Weyl approach . . . . . . . . . . . . 5

2.3 Functional calculus: The Riesz approach . . . . . . . . . . . . 6

$\begin{array}{lll}3 & \text { Riesz-Clifford Calculus for Non-Commuting Operators } & 8\end{array}$

3.1 Clifford Algebras and Clifford Analysis . . . . . . . . . . . 8

3.2 Riesz-Clifford calculus: a Polynomial Procedure . . . . . . . . . 11

3.3 Riesz-Clifford Calculus: an Integral Representation Procedure 13

$4 \quad$ Riesz-Clifford Calculus and Quantum Field Theory 16

4.1 Quantum Field Theory and Clifford Algebras . . . . . . . . . 16

4.2 Quantum Field Theory and Riesz-Clifford Calculus . . . . . . 17

\section{Introduction}

The quantum mechanical connection was certainly the strongest of the motivations for the development of operator algebra.

Irving E. Segal, 26]

The quantization procedure [3], [5], [13], [17] requires the construction of some mapping from an algebra of real valued functions (the algebra of classic observables) to an algebra of self-adjoint operators (the algebra of quantum observables). Analogously, one should be able (in some sense) to construct functions from self-adjoint (non-commuting) operators. The importance of this problem in the physics and the non-triviality of the corresponding mathematical problems has been amply illustrated during the last 50 years (in addition to the above mentioned papers see also recent ones [11], [24], [26] and their rich bibliographies).

The problem of quantization may be reformulated mathematically as a problem on construction of a functional calculus. The already classical holomorphic calculus, which is based on analysis of several complex variables, allows only the situation of mutually commuting operators (see the milestone papers of J. L. Taylor [29], [30]). Other approaches also lead only to 
the commuting case (see, for example, 23]). Such constructions do not meet the needs of quantum mechanics, where non-commutativity of observables is a principal assumption.

The main goal of the present paper is a construction of a Clifford hyperholomorphic calculus of several non-commuting operators and to show its applicability to quantum field theory.

It should be mentioned that functional calculus may be considered at two different levels. The first one is the original problem of quantum mechanics of constructing functions from the operators of coordinates $Q_{j}$ and impulses $P_{j}$, which satisfy the well known Heisenberg commutation relations

$$
\left[Q_{j}, P_{i}\right]=i \hbar \delta_{i j} I
$$

In this context there are many different approaches to quantization with a large number of nice results (for example, the PDO calculus [13], [14], [27], [31] and the Toeplitz operators in the Segal-Bargman space [5], [6]). The richness of the calculus is rooted in the structure of the Heisenberg group [15], [16]. At a second level one can construct a functional calculus from an arbitrary finite set of self-adjoint operators $\left\{T_{j}\right\}, 1 \leq j \leq m$. In this case the number of possible quantizations and the precise results obtained is much smaller [22] and sometimes only a more-or-less formal power series may be established in such a setting. In [18 it was shown that one can preserve the essential features at the first level if the assumption is made, that the operators $\left\{T_{j}\right\}, 1 \leq j \leq m$ represent some Lie algebra $\mathfrak{g}$.

In Section 2 of this work we discuss general properties and known results of functional calculus. In Section 3 we construct the Riesz-like hyperholomorphic functional calculus for a set of non-commuting operators based on Clifford analysis. To extend the calculus from commuting operators to non-commuting ones we restrict the family of functions from smooth ones to hyperholomorphic functions. It should be noted that hyperholomorphic functions do not form an algebra (at least under usual multiplication) and this is reflected at Definition 3.6 of Riesz-Clifford calculus. In Section 1 it is shown that the Riesz-Clifford functional calculus is a relevant model in quantum field theory corresponding to the Weyl calculus in quantum mechanics.

We consider only the case of self-adjoint operators on the Hilbert space $H$ (or of elements in an abstract $C^{*}$-algebra). It is also possible to consider the more general setting of self-adjoint operators in a Banach space [2] (or of elements in an abstract Banach algebra). 
We are grateful to V. V. Kravchenko, J. Ryan, and I. Spitkovsky for their valuable comments.

\section{Different Approaches to Functional Calcu- lus}

In this Section we give a short overview of different approaches to functional calculus.

\subsection{Two Procedures of Functional Calculus}

The following definition is preliminary.

Definition 2.1 A functional calculus $\Phi(\mathfrak{A}, T)$ for an $m$-tuple of self-adjoint operators $T=\left(T_{1}, \ldots, T_{m}\right)$ on the Hilbert space $H$ is a continuous mapping $\Phi: \mathfrak{A} \rightarrow \mathcal{B}(H)$ of a linear space $\mathfrak{A}$ of functions into a linear space of operators on $H$ with some natural properties.

We do not specify yet the algebra $\mathfrak{A}$ and the natural properties. In many cases the algebra $\mathfrak{A}$ should at least contain all smooth functions with compact support (space $C_{c}^{\infty}(\Omega)$ ) in set $\Omega \subset \mathbb{R}^{m}$ depending of the spectra of the operators $T_{j}$. The natural conditions usually include some combinations of the following items:

1. $\Phi(1)=I$, i. e., the image of the function identically equal to 1 is the identity operator on $H$;

2. $\Phi(\bar{f})=\Phi(f)^{*}$;

3. $\Phi\left(x_{j}\right)=T_{j}$

4. $\|\Phi(f)\|_{H} \leq\|f\|_{\mathfrak{A}}$

5. $\Phi(\mathfrak{A}, T)$ depends continuously on the set $T$.

It is notable, that the algebraic properties 1-3 usually imply the metric ones 4,5 .

Generally speaking there are two different but closely connected ways to construct a functional calculus. 
Procedure 2.2 The homomorphism $\Phi$ is defined on some dense subalgebra $\mathfrak{A}_{0}$ of the algebra $\mathfrak{A}$. The algebra $\mathfrak{A}_{0}$ should have a simple algebraic structure (for example, the algebra of polynomials), which allows a simple definition of $\Phi$. After that $\Phi$ extends to the whole of $\mathfrak{A}$ by continuity.

Procedure 2.3 Consider a reproducing formula for functions from $\mathfrak{A}$ of the form

$$
f(x)=\int K(x, y) f(y) d y \text {. }
$$

For example, a Cauchy type formula (see Subsection 2.3) or a Fourier type transform (see Subsection 2.2). If the kernel $K(x, y)$ can be extended in a natural way to an operator $K(T, y)$, then we define the functional calculus by the formula

$$
\Phi(f)=f(T)=\int K(T, y) f(y) d y .
$$

The first Procedure is sometimes easier, but unlike the second one it does not give us an explicit formula for functions outside $\mathfrak{A}_{0}$. Usually it is possible to construct, the function calculus in both ways (see examples bellow). The first Procedure is carried out for the holomorphic calculus in [30] and the second one in [29].

\subsection{Functional calculus: The Weyl approach}

Let us recall that a PDO Op $a(x, \xi)$ [14], [27], [31], [33], with the Weyl symbol $a(x, \xi)$, is defined by the formula:

$$
[\mathrm{Op} a](x, \xi) u(y)=\int_{\mathbb{R}^{n} \times \mathbb{R}^{n}} a\left(\frac{x+y}{2}, \xi\right) e^{i\langle y-x, \xi\rangle} u(x) d x d \xi
$$

This formula may be obtained by Procedure 2.3 from the formula of the inverse Fourier transform

$$
f(x)=(2 \pi)^{-n / 2} \int_{\mathbb{R}^{n}} \widehat{f}(\xi) e^{i x \xi} d \xi .
$$

Namely, let us take the set of self-adjoint operators

$$
T_{j}=y_{j}, T_{j+n}=\frac{1}{i} \frac{\partial}{\partial y_{j}}, 1 \leq j \leq n
$$


which operate on $S=\mathbb{R}^{N}$ in the obvious way. Note that these operators have exactly the commutators (11). Then we have (see [32], § 1.3)

$$
\begin{aligned}
{[K f](y) } & =(2 \pi)^{-N} \int_{\mathbb{R}^{2 N}} \widehat{k}(x, \xi) e^{i\left(\sum_{1}^{N} x_{j} y_{j}-\sum_{1}^{N} \xi \frac{\partial}{i \partial y_{j}}\right)} f(y) d x d \xi \\
& =(2 \pi)^{-N} \int_{\mathbb{R}^{2 N}} k\left(\frac{x+y}{2}, \xi\right) e^{i\langle y-x, \xi\rangle} f(x) d x d \xi
\end{aligned}
$$

i. e. it defines exactly the Weyl functional calculus (or the Weyl quantization). PDO calculus is a very important tool for the theory of differential equations and quantum mechanics.

REMARK 2.4 Feynman proposed in [12] an extension of this functional calculusthe functional calculus of ordered operators - in a very similar way. Anderson [2] introduced a generalization of the Weyl calculus for an arbitrary set $\left\{T_{j}\right\}$ of self-adjoint operators in a Banach space by the formula

$$
K=(2 \pi)^{-N / 2} \int_{\mathbb{R}^{N}} \widehat{k}\left(x_{1}, x_{2}, \ldots, x_{N}\right) e^{i \sum_{1}^{N} x_{j} T_{j}} d x,
$$

A description of several different operator calculus may be found in 22]. It was shown by R. Howe [15], [16] that the success of the original Weyl calculus is intimately connected with the structure of the Heisenberg group and its different representations.

REMARK 2.5 It should be noted, that besides the definition of the Weyl functional calculus in accordance with the Procedure 2.3 it can be defined also by the Procedure 2.2. As subalgebra $\mathfrak{A}_{0}$ should be taken the algebra of symmetric polynomials of the variables $x_{j}$ and the homomorphism $\Phi$ "insert" the operators $T_{j}$ instead of the variables $x_{j}$ [2], Theorem 2.4. We will use this observation to establish connection between the Weyl and the Riesz-Clifford calculus.

\subsection{Functional calculus: The Riesz approach}

For a pair of self-adjoint operators $T_{1}, T_{2}$, the Riesz calculus [25], Chap. XI can be also developed in accordance with the Procedure 2.3. The starting point is the Cauchy integral formula for a function $f(z)$ holomorphic in $z=$ 
$x_{1}+i x_{2}$ near the spectrum $\sigma(T)$ of the operator $T=T_{1}+i T_{2}$ and a nice contour $\Gamma$ containing $\sigma(T)$ :

$$
f\left(x_{1}+i x_{2}\right)=(2 \pi)^{-1} \int_{\Gamma} f(\tau)\left(\tau-\left(x_{1}+i x_{2}\right)\right)^{-1} d \tau .
$$

Thus if we are able to define the function $K(z, \tau)=\left(\tau I-\left(x_{1}+i x_{2}\right)\right)^{-1}$ for an operator $T=T_{1}+i T_{2}$, then the functional calculus will be given by the formula

$$
f\left(T_{1}+i T_{2}\right)=(2 \pi)^{-1} \int_{\Gamma} f(\tau)\left(\tau I-\left(T_{1}+i T_{2}\right)\right)^{-1} d \tau .
$$

By Runge's theorem, the Riesz calculus may also be obtained by Procedure 2.2 as the closure of the polynomials in $T$ in the space of holomorphic functions. This definition, as was shown in [2], Theorem 5.1, gives that the Riesz and the Weyl calculus are essentially the same in the case of a pair of bounded operators $\left(T_{1}, T_{2}\right)$.

To generalize the Riesz calculus from a pair of operators to arbitrary $m$ tuples, one should use a generalization of complex analysis in one variable. There are many essentially different theories which can be considered as generalizations. The principal contributions to holomorphic functional calculus based on the analysis of several complex variables were made in [29], [30]. By the very nature of complex analysis, this approach can work only for a commuting set of operators.

Another approach to the Riesz calculus based on Clifford analysis may be found in 23], but there again it was found that such a functional calculus is possible only for an m-tuple of commuting generalized scalar operators with real spectra [23], Theorem 9.1.

Despite of the rich mathematical aspects of these theories, it should be noted that quantum mechanics requires a calculus of essentially noncommuting operators. Moreover, by the von Neumann theorem [1], § 90 it follows, that for any $m$-tuple $\left\{T_{j}\right\}$ of commuting self-adjoint operators, there are such $m$-tuple of real valued functions $\left\{f_{j}\right\}$ and an operator $S$ such that

$$
T_{j}=f_{j}(S)
$$

Thus a calculus for $m$-tuple of commuting operators is (in some sense) the calculus of only one operator. Therefore one is forced to look for another definition of a non-trivial functional calculus of a set of non-commuting operators. 


\section{Riesz-Clifford Calculus for Non-Commuting Operators}

To extend the Riesz calculus to an arbitrary $m$-tuples of bounded operators $\left\{T_{j}\right\}$ it seems natural to use Clifford analysis (see for example [8]), which is a non-commutative analogy to one-dimensional complex analysis. Then one can define a function from an arbitrary $m$-tuple of bounded self-adjoint operators $\left\{T_{j}\right\}$, either by the Cauchy integral formula (compare with (7) and [18], Remark 4.11) or by the subset of symmetric hyperholomorphic polynomials [21].

In this Section we will systematically develop such a point of view.

\subsection{Clifford Algebras and Clifford Analysis}

We need some notation from [8]. Let the euclidean vector space $\mathbb{R}^{n+1}$ have the orthonormal basis $e_{0}, e_{1}, \ldots, e_{n}$. The Clifford algebra $\mathbf{C l}(n, 0)$ is generated by the elements $e_{0}, e_{1}, \ldots, e_{n}$ with the usual vector operations and the multiplication defined on the elements of the basis by the following equalities

$$
\begin{aligned}
e_{j}^{2} & =-e_{0} \quad(j=1, \ldots, n), \\
e_{j} e_{k}+e_{k} e_{j} & =0 \quad(j, k=1, \ldots, n ; j \neq k),
\end{aligned}
$$

and then extended linearly to the whole space. An element of $\mathbf{C l}(n, 0)$ can be written as a linear combination with coefficients $a_{\alpha} \in \mathbb{R}$ of the monomials $e_{\alpha}=e_{1}^{j_{1}} e_{2}^{j_{2}} \cdots e_{m}^{j_{m}}$ :

$$
a=\sum_{\alpha} a_{\alpha} e_{\alpha}=\sum_{j_{k}=0 \text { or } 1} a_{j_{1} j_{2} \ldots j_{m}} e_{1}^{j_{1}} e_{2}^{j_{2}} \cdots e_{m}^{j_{m}} .
$$

The conjugate $\bar{a}$ of an element $a$ is defined by the rule:

$$
\bar{a}=\sum_{\alpha} a_{\alpha} \bar{e}_{\alpha}=\sum_{j_{k}=0 \text { or } 1} a_{j_{1} j_{2} \ldots j_{m}} \bar{e}_{m}^{j_{m}} \bar{e}_{m-1}^{j_{m-1}} \cdots \bar{e}_{1}^{j_{1}},
$$

where $\bar{e}_{j}=-e_{j}, \bar{e}_{0}=e_{0}, 1 \leq j \leq n$. A Clifford algebra valued function $f$ of the variables $\left(x_{0}, x_{1}, \ldots, x_{n}\right)$, is called hyperholomorphic in an open domain $\Omega \subset \mathbb{R}^{n+1}$ if it satisfies the Dirac (or Weyl $\mathbb{8}$ ) equation

$$
D f=\sum_{j=0}^{n} e_{j} \frac{\partial f}{\partial x_{j}}=0 .
$$


The main results of Clifford analysis (Cauchy theorem, Cauchy integral formula etc.) have a structure closer that of the complex analysis of one variable than to standard complex analysis of several variables.

Note one very important feature. In Clifford analysis not all polynomials are hyperholomorphic functions. Instead one has to consider the symmetric polynomials of the monomials having the form

$$
\vec{x}_{j}=\left(e_{j} x_{0}-e_{0} x_{j}\right), 1 \leq j \leq n .
$$

The role of such monomials ("regular variables" [7]) is described for quaternionic analysis in [28], for Clifford analysis in [21], for Fueter-Hurwitz analysis in [19], and for solutions of the general Dirac type equation in [18], [20].

We use the following facts and notation. Let $H(\Omega)$ denote the space of all hyperholomorphic functions in the domain $\Omega$ and by $P$ the space of all hyperholomorphic polynomials. The space $P$ has the linear subspaces $P_{j}, 0 \leq j<\infty$ of homogeneous polynomials degree $j$. We will show that $P$ consist of symmetric polynomials constructed from the monomials of the form (11) by symmetric products [21]

$$
a_{1} \times a_{2} \times \cdots \times a_{k}=\frac{1}{k !} \sum a_{j_{1}} a_{j_{2}} \cdots a_{j_{k}}
$$

where the sum is taken over all of permutations of $\left(j_{1}, j_{2}, \ldots, j_{k}\right)$. Clifford valued coefficients are written on the right-hand side.

Lemma 3.1 The linear subspace $P_{j}$ has a basis consisting of symmetric polynomials.

Proof. Let $V_{\alpha}(x)$ be the following homogeneous hyperholomorphic polynomial of degree $|\alpha|=k$ (see [8], Chap. II, Definition 1.5.1):

$$
V_{\alpha}(x)=\sum_{j=0}^{|\alpha|} \frac{(-1)^{j} x_{0}^{j}}{j !}\left[\left(\sum_{l=1}^{n} \frac{\partial}{\partial x_{l}}\right)^{j}\left(\sum_{l=1}^{n} x_{l}\right)^{\alpha}\right] .
$$

Then, the $V_{\alpha}$ with $|\alpha|=k$, form a basis of $V_{k}$. It is easy to check that

$V_{\alpha}(x)=(-1)^{\alpha_{1}}\left(e_{0} x_{1}-e_{1} x_{0}\right)^{\alpha_{1}} \times\left(e_{0} x_{2}-e_{2} x_{0}\right)^{\alpha_{2}} \times \cdots \times\left(e_{0} x_{n}-e_{n} x_{0}\right)^{\alpha_{n}}=\vec{x}^{\alpha}$.

This gives the assertion. 
Let

$$
E(y-x)=\frac{\Gamma\left(\frac{n+1}{2}\right)}{2 \pi^{(m+1) / 2}} \frac{\overline{y-x}}{|y-x|^{n+1}}
$$

be the Cauchy kernel [8], p. 146 and

$$
d \sigma=\sum_{j=0}^{n}(-1)^{j} e_{j} d x_{0} \wedge \ldots \wedge\left[d x_{j}\right] \wedge \ldots \wedge d x_{m} .
$$

be the differential form of the "oriented surface element" [8], p. 144. Then for any $f(x) \in H(\Omega)$ we have the Cauchy integral formula [8], p. 147

$$
\int_{\partial \Omega} E(y-x) d \sigma_{y} f(y)=\left\{\begin{array}{l}
f(x), x \in \Omega \\
0, x \notin \bar{\Omega}
\end{array} .\right.
$$

We should point out the universality (with respect to domains) of both the Cauchy kernel $E(y-x)$ and the Cauchy formula in the Clifford analysis, in contrast to the case of several complex variables.

If we define as in [4], $\S 18.6$ and [8], Chap. II, Definition 1.5.5

$$
W_{\alpha}^{(a)}(x)=(-1)^{|a|} \partial^{\alpha} E(x-a),
$$

then, for $|x|<|y|$ we obtain [8], Chap. II, (1.16)

$$
E(y-x)=\sum_{j=0}^{\infty}\left(\sum_{|\alpha|=j} V_{\alpha}(x) W_{\alpha}(y)\right) .
$$

Let $M_{(r)}(\Omega)$ be the unitary right Clifford module of all left hyperholomorphic functions in $\Omega$. For fixed $a R_{(r)}(a)$ denotes the right Clifford module generated by functions $W_{\alpha}^{(a)}(x)$. If $S=\left\{a_{i} \mid i \in \mathbb{N}\right\}$ is a finite or countable subset of $\mathbb{R}^{n+1}$, put $R_{(r)}(S)=\cup_{i \geq 1} R_{(r)}\left(a_{i}\right)$.

The following result plays an important role latter.

Theorem 3.2 (Runge type) [4], $\S 18$ Let $K$ be a compact subset of $\mathbb{R}^{n+1}$ and let $S$ be a subset of $\mathbb{R}^{n+1} \backslash K$ having one point in each bounded component of $\mathbb{R}^{n+1} \backslash K$. The $n$ each function which is hyperholomorphic in a neighborhood of $K$ can be approximated uniformly on $K$ by the functions in $M_{(r)}\left(\mathbb{R}^{n+1}\right) \oplus R_{(r)}(S)$, i. e. $M_{(r)}\left(\mathbb{R}^{n+1}\right) \oplus R_{(r)}(S)$ is uniformly dense in $H(K)$. 


\subsection{Riesz-Clifford calculus: a Polynomial Procedure}

Fix an $m$-tuple of bounded self-adjoint operators $T=\left(T_{1}, \ldots, T_{m}\right)$ on the Hilbert space $H$. It is well known [8], Chap. I that any Clifford algebra (particularly, $\mathbf{C l}(n, 0)$ ) can be realized as an algebra of endomorphisms of some finite-dimensional vector space $H_{0}$. Let $\widetilde{H}=H \otimes H_{0}$. Then if $n \geq m$ we can associate with $T$ an operator $\widetilde{T}: \widetilde{H} \rightarrow \widetilde{H}$ by the formula

$$
\widetilde{T}=\sum_{j=1}^{m} T_{j} \otimes e_{j} .
$$

We use the notation $\mathcal{B}(H)$ and $\mathcal{B}(\widetilde{H})$ for the algebra of bounded operators in the corresponding Hilbert space.

Definition 3.3 Let $\mathfrak{A}$ be an algebra with the operations of addition $+_{\mathfrak{A}}$ and multiplication $\cdot \mathfrak{A}$ which is generated by a finite set of elements $a_{1}, \ldots, a_{k}$. Define a new operation $\times_{\mathfrak{A}}$ of symmetric multiplication associated with them by the formula (12). The resulting set will be called an $\times$-algebra. Let $\mathfrak{A}$ and $\mathfrak{B}$ be two $\times$-algebras. We say that $\phi: \mathfrak{A} \rightarrow \mathfrak{B}$ is an $\times$-homomorphism of two $\times$-algebras if the following holds

1. $\phi\left(a_{j}\right)=b_{j}, 1 \leq j \leq k$, where $a_{1}, \ldots, a_{k}$ and $b_{1}, \ldots, b_{k}$ are generators of $\mathfrak{A}$ and $\mathfrak{B}$ correspondingly.

2. $\phi\left(\lambda a_{1}+a_{2}\right)=\lambda \phi\left(a_{1}\right)+\phi\left(a_{2}\right)$ for any $a_{1}, a_{2} \in \mathfrak{A}$ and $\lambda \in \mathbb{C}$.

3. $\phi\left(a_{1} \times \cdots \times a_{n}\right)=\phi\left(a_{1}\right) \times \cdots \times \phi\left(a_{n}\right)$ for any set $a_{1}, \ldots, a_{n}$ of (not necessarily distinct) generators of $\mathfrak{A}$.

By the definition of the symmetric product one can obviously deduce

Lemma 3.4 Any homomorphism of two algebras is a $\times$-homomorphism of the corresponding $\times$-algebras.

The converse, of course, is not true:

Example 3.5 Let $\mathfrak{A}$ be the algebra of polynomials in two variables $x_{1}$ and $x_{2}$, and $\mathfrak{B}$ be the algebra generated by two operators $P$ and $Q$ with the commutator $[P, Q]=i \hbar I$. Then the mapping $\phi$ defined on the generators of algebra $\mathfrak{A}$ by

$$
\phi: x_{1} \mapsto P, \phi: x_{2} \mapsto Q
$$


cannot be extended to an homomorphism of algebras $\mathfrak{A}$ and $\mathfrak{B}$ but defines a $\times$-homomorphism, which coincides with the Weyl quantization.

The following is a definition of Riesz-Clifford hyperholomorphic calculus (compare with [23]).

Definition 3.6 We say that $\widetilde{T}$ has a functional calculus $(\mathfrak{A}, \Phi)$ based on $\mathbb{R}^{m}$ whenever the following conditions hold: $\mathfrak{A}$ is a topological vector space of hyperholomorphic functions from $\mathbb{R}^{m}$ to $\mathbf{C l}(0, n)(m \leq n)$, with addition and (symmetric) multiplication defined pointwise, and $\Phi: \mathfrak{A} \rightarrow \mathcal{B}(\widetilde{H})$ is a continuous mapping and its restriction to symmetric polynomials is a $\times$ homomorphism such that

$$
\Phi: \vec{x}_{j}\left(=e_{j} x_{0}-e_{0} x_{j}\right) \mapsto T_{j}, 1 \leq j \leq m
$$

There, to extend calculus from commuting operators to non-commuting we ones we relax the requirement from homomorphism to $\times$-homomorphism.

Definition 3.7 The support of the homomorphism $\Phi$ is the smallest closed set in $\mathbb{R}^{n}$ such that $\Phi(f)=0$ for all $f \in \mathfrak{A}$ with $\operatorname{supp} \Phi \cap \operatorname{supp} f=\emptyset$. The joint $\times$-resolvent set $R_{\times}(T)$ of $T$ is the largest open subset of $\mathbb{R}^{m}$ such that for any point $\lambda=\left(\lambda_{1}, \ldots, \lambda_{m}\right) \in R_{\times}(T)$, the $\times$-algebra generated by the operators $T_{j} \otimes e_{j}-\lambda_{j} I \otimes e_{j}, 1 \leq j \leq m$ contains at least one invertible element in $\widetilde{H}$. The joint spectrum $\sigma_{\times}(T)$ of the operators $T_{1}, \ldots, T_{m}$ is the set $\mathbb{R}^{m} \backslash \sigma_{\times}(T)$.

Theorem 3.8 (Uniqueness) In the case of $n=m$ for a given simplyconnected domain $\Omega$ and an $m$-tuple of operators $T$, there exists no more than one hyperholomorphic functional calculus.

ProOF. By the $\times$-homomorphism conditions one can extend the correspondence (20) to the space $P$ of hyperholomorphic polynomials in only one way. Then the denseness of $P$ in $H(\Omega)$ implies the assertion.

Theorem 3.9 For any $m$-tuple $T$ of bounded self-adjoint operators there exist a hyperholomorphic calculus on $\mathbb{R}^{m}$. 
Proof. Any hyperholomorphic function on $f(x) \in H\left(\mathbb{R}^{m}\right)$ can be represented as the Taylor series [8], Chap. II, $\S 1.6 .3$, Remark 2

$$
f(x)=\sum_{|\alpha|=1}^{\infty} V_{\alpha}(x) c_{\alpha}, \quad c_{\alpha} \in \mathbf{C l}(n, 0) .
$$

We have already seen what is $V_{\alpha}(T)$, thus the functional calculus may be defined by the formula

$$
f(T)=\sum_{|\alpha|=1}^{\infty} V_{\alpha}(T) c_{\alpha} .
$$

The convergence of this series is evident.

\subsection{Riesz-Clifford Calculus: an Integral Representa- tion Procedure}

We now deduce the explicit formula for hyperholomorphic calculus. Accordingly to Procedure 2.3 we should define the Cauchy kernel of the operators $T_{j}$ as follows.

Definition 3.10 Let $n \geq m$. Then (cf. 19)

$$
E(y, T)=\sum_{j=0}^{\infty}\left(\sum_{|\alpha|=j} V_{\alpha}(T) W_{\alpha}(y)\right)
$$

where

$$
V_{\alpha}(T)=T_{1}^{\alpha_{1}} \times \cdots \times T_{m}^{\alpha_{m}} \times I \times \cdots \times I ;
$$

i. e., we have formally substituted in (14) for $\left(\vec{x}_{1}, \ldots, \vec{x}_{n}\right)$ the $n$-tuple of operators $\left(T_{1}, \ldots, T_{m}, I, \ldots, I\right)$.

REMARK 3.11 For the sake of the simplicity we will consider in this Section only the case $m=n$. Consideration of the more general case $n \geq m$ is analogous and will be needed Section 4 .

Lemma 3.12 Let $|T|=\max _{j}\left\{\left\|T_{j}\right\|\right\}$. Then for fixed $|y|>|T|$, the equation (21) defines a bounded operator in $\widetilde{H}$. 
Proof. Consideration of the real valued series

$$
\begin{aligned}
\|E(y, T)\| & \leq \sum_{j=0}^{\infty}\left(\sum_{|q|=j}\left\|V_{\alpha}(T)\right\|\left\|W_{\alpha}(y)\right\|\right) \\
& \leq \sum_{j=0}^{\infty}\left(\sum_{|a|=j}|T|^{j}\left\|W_{\alpha}(y)\right\|\right),
\end{aligned}
$$

and the properties of the Cauchy kernels shows that for the mentioned $y$ the series (21) converges in the uniform operator topology to a bounded operator.

Definition 3.13 The maximal open subset $R_{C}(T)$ of $\mathbb{R}^{n}$ such that for any $y \in R_{C}(T)$ the series in (21) converges in the uniform operator topology to a bounded operator on $\widetilde{H}$ will be called the (Clifford) resolvent set of $T$. The complement of $R_{C}(T)$ in $\mathbb{R}^{n}$ will be called the (Clifford) spectral set of $T$ and denoted by $\sigma_{C}(T)$.

From Lemma 3.12 it follows that $R_{C}(T)$ is always non- empty and from Definition 3.13 one can see that $\sigma_{C}(T)$ is closed. Moreover, it is easy to see that

Lemma 3.14 The Clifford spectral set $\sigma_{C}(T)$ is compact.

Proof. $\sigma_{C}(T)$ is a closed subset of $\mathbb{R}^{n}$ which is bounded due to Lemma 3.12.

Lemma 3.15 Let $r>|T|$ and let $\Omega$ be the ball $\mathbb{B}(0, r) \in \mathbb{R}^{m}$. Then for any symmetric polynomial $P(\vec{x})$ we have

$$
P(T)=\int_{\partial \Omega} E(y, T) d \sigma_{y} P(y)
$$

where $P(T)$ is the symmetric polynomial of the $m$-tuple $T$.

Proof. First, consider the case of the polynomial $P(\vec{x})=V_{\alpha}(\vec{x})$. Recall the formula [8], Chap. II, Lemma 1.5.7(i)

$$
\int_{\partial \mathbb{B}(0, r)} W_{\beta}(y) d \sigma V_{\alpha}(y)=\delta_{\alpha \beta} .
$$


Let $A$ be an operator defined by the right-hand side of (23), since the convergence in (21) is uniform, we obtain

$$
\begin{aligned}
A & =\int_{\partial \Omega} E(y, T) d \sigma_{y} P(y) \\
& =\int_{\partial \Omega} E(y, T) d \sigma_{y} V_{\alpha}(y) \\
& =\int_{\partial \Omega} \sum_{j=0}^{\infty}\left(\sum_{|q|=j} V_{\beta}(T) W_{\beta}(y)\right) d \sigma_{y} V_{\alpha}(y) \\
& =\sum_{j=0}^{\infty}\left(\sum_{\mid q=j} V_{\beta}(T) \int_{\partial \Omega} W_{\beta}(y) d \sigma_{y} V_{\alpha}(y)\right) \\
& =\sum_{j=0}^{\infty}\left(\sum_{|\sigma|=j} V_{\beta}(T) \delta_{\alpha \beta}\right) \\
& =V_{\alpha}(T) \\
& =P(T) .
\end{aligned}
$$

Finally, by linear extension we get the assertion.

Lemma 3.16 For any domain $\Omega$ which does not contain $\sigma_{C}(T)$ and any $f \in H(\Omega)$, we have

$$
\int_{\partial \Omega} E(y, T) d \sigma_{y} f(y)=0
$$

Proof. If we apply a bounded linear functional $w$ on the space $\mathcal{B}(\widetilde{H})$ to the integral at left-hand side of the assertion, we will obtain an integral of the hyperholomorphic function

$$
w(E(y, T)) d \sigma_{y} f(y),
$$

which is equal to 0 by the Cauchy theorem. The arbitrariness of $w$ gives the assertion.

Due to this Lemma we can replace the domain $\mathbb{B}(0, r)$ at Lemma 3.15 with an arbitrary domain $\Omega$ containing the spectral set $\sigma_{C}(T)$. An application of Lemma 3.15 gives the main 
Theorem 3.17 Let $T=\left(T_{1}, \ldots, T_{m}\right)$ be an $m$-tuple of bounded self-adjoint operators. Let the domain $\Omega$ with piecewise smooth boundary have a connected complement and suppose the spectral set $\sigma_{C}(T)$ lies inside a domain $\Omega$. Then the mapping

$$
\Phi: f(x) \mapsto f(T)=\int_{\partial \Omega} E(y, T) d \sigma_{y} f(y)
$$

defines a hyperholomorphic calculus for $T$.

Proof. We have already seen (Lemma 3.15) that formula (26) defines a functional calculus for functions in $M_{(r)}\left(\mathbb{R}^{n+1}\right)$. Application of Theorem 3.2 allows us to extend this calculus to the whole of $H(\Omega)$.

\section{Riesz-Clifford Calculus and Quantum Field Theory}

\subsection{Quantum Field Theory and Clifford Algebras}

The step from a quantum system with one particle to quantum ensemble with $m$ particles depends on the kind of particles we have. For example, photons satisfy to Bose-Einstein statistics, but electrons satisfy to FermiDirac statistics [9]. This means that observables of a system of photons are symmetrical operators relative to equivalent particles; but observables of a system of electrons are anti-symmetric ones. This involves, in particular, the Pauli's exclusion principle: there is only one or no Fermi particle in each state. Such a distribution of particles among different states can be easily described with the help of the Clifford algebra $\mathbf{C l}(n, 0)$. The monomial $e_{1}^{i_{1}} e_{2}^{i_{2}} \cdots e_{m}^{i_{m}}$ can be considered as the description of a physical system, in which the only states enumerated by $k$, for which $i_{k} \neq 0$, are filled by a Fermi particle. The general element

$$
a=\sum_{\alpha} a_{\alpha} e_{\alpha}=\sum_{j_{k}=0 \text { or } 1} a_{j_{1} j_{2} \ldots j_{m}} e_{1}^{j_{1}} e_{2}^{j_{2}} \cdots e_{m}^{j_{m}}
$$

of $\mathbf{C l}(m, 0)$ can be interpreted as a probability distribution among "pure" states defined by such monomials [9].

\footnotetext{
${ }^{1}$ We consider only the case of a finite number of different states. The difficulties occurred in the infinite case is described for example in 26].
} 


\subsection{Quantum Field Theory and Riesz-Clifford Calcu- lus}

Non-commutativity of quantum observables generate the following question: Let $A$ and $B$ be quantum analogues of classic observables $a$ and $b$. What corresponds to the classic observable ab, the result of simultaneous measurement of $a$ and $b$ ?

Jordan (see for example [17], § 1.2) proposed the answer

$$
A \circ B=\frac{1}{2}(A B+B A)=\left(\frac{A+B}{2}\right)^{2}-\left(\frac{A-B}{2}\right)^{2} .
$$

One can interpret this formula in the following way. Simultaneous measurement of $A$ and $B$ from the macroscopic point of view is not simultaneous from the microscopic one. It is natural to assume that two different processes (measurement of $A B$ and $B A$ ) will occur with equal probability $\frac{1}{2}$. This is explicitly described fy by (27).

To prolong such a point of view for three operators $A, B, C$ we can accept neither $(A \circ B) \circ C$ nor $A \circ(B \circ C)$ (the Jordan multiplication (27) is not associative!). We again should select the equal probability $\frac{1}{6}$ for six different processes $A B C, A C B, B A C, B C A, C A B, C B A$ and take the value

$$
\frac{1}{6}(A B C+A C B+B A C+B C A+C A B+C B A)=A \times B \times C .
$$

Note also that $A \circ B=A \times B$.

Prolonging this line we should agree that the symmetric monomial

$$
A_{1} \times A_{2} \times \cdots \times A_{k}
$$

correspond to the monomial of classic observables

$$
a_{1} \times a_{2} \times \cdots \times a_{k} .
$$

Thus the symmetric polynomials are a quantum version of classic polynomials. Then the natural condition of continuity leads us to the Riesz-Clifford functional calculus.

\footnotetext{
${ }^{2}$ In spite of some advantages of the Jordan algebras, they were not too successful and have almost disappeared in the modern literature.
} 
One can summarize the main point of this Section the following way: To describe of an ensemble of Fermi particles we have to study the algebra of operators on the space $\widetilde{H}=H \otimes H_{0}$, where the Hilbert space $H$ is a space of states of one particle and $H_{0}$ is a (finite-dimensional in our consideration) space of filling numbers. The Riesz-Clifford functional calculus based on the symmetric product naturally describes functions of non-commuting quantum observables in quantum field theory.

\section{References}

1. N. I. Akhiezer and I. M. Glazman. Theory of Linear Operators in Hilbert Space, volume 1. Pitman Advanced Publishing Program, London, 1981.

2. R. F. V. Anderson. The Weyl functional calculus. J. Funct. Anal., 4:240-267, 1969.

3. F. A. Berezin. Quantization. Math. USSR Izvestija, 8:1109-1165, 1974.

4. F. Brackx, R. Delanghe, and F. Sommen. Clifford Analysis, volume 76 of Research Notes in Mathematics. Pitman Advanced Publishing Program, Boston, 1982.

5. L. A. Coburn. Berezin-Toeplitz quantization. In Algebraic Mettods in Operator Theory, pages 101-108. Birkhäuser, New York, 1994.

6. L. A. Coburn and J. Xia. Toeplitz algebras and Rieffel deformation. Comm. Math. Phys., 1994. to appear.

7. R. Delanghe. On the singularities of functions with values in a Clifford algebra. Math. Ann. 196(1972), pages 293-319.

8. R. Delanghe, F. Sommen, and V. Souček. Clifford Algebra and SpinorValued Functions. Kluwer Academic Publishers, Dordrecht, 1992.

9. P. A. M. Dirac. Lectures on Quantum Field Theory. Yeshiva University, New York, 1967.

10. R. Doran, editor. $C^{*}$-Algebras: 1943-1993. Number 167 in Contemporary Mathematics. AMS, Providence, Rhode Island, 1994. 
11. E. G. Effros. Some quantizations and reflections inspired by the GelfandNaimark theorem. In Doran [10], pages 99-113.

12. R. P. Feynman. An operator calculus having applications in quantum electrodynamics. Phys. Rev., 84(2):108-128, 1951.

13. G. B. Folland. Harmonic Analysis in Phase Space. Princeton University Press, Princeton, New Jersey, 1989.

14. L. Hörmander. The Analysis of Linear Partial Differential Operators III: Pseudodifferential Operators. Springer-Verlag, Berlin Heidelberg New York Tokyo, 1985.

15. R. Howe. On the role of the Heisenberg group in harmonic analysis. Bull. of the AMS (New Series), 3(2):821-843, 1980.

16. R. Howe. Quantum mechanics and partial differential equations. $J$. Funct. Anal., 38:188-254, 1980.

17. A. A. Kirillov. Geometric Quantization, volume 4 of Encyclopedia of Mathematical Sciences, pages 137-172. Springer-Verlag, Berlin, 1990.

18. V. V. Kisil. Relative convolutions. I. Properties and applications. Reporte Interno \# 162, Departamento de Matemáticas, CINVESTAV del I.P.N., Mexico City, 1994 (to appear in Advances in Mathematics).

19. W. Królikowski and E. Ramírez de Arellano. Polynomial solutions of the Fueter-Hurwitz equation. In A. Nagel and E. L. Stout, editors, The Madison Symposium on Complex Analysis, number 137 in Contemprorary Mathematics, pages 297-305. AMS, Providence, Rhode Island, 1992.

20. G. Laville. Sur un calcul symbolique de Feynmann. In Seminar d'Analyse, volume 1295 of Lect. Notes in Math., pages 132-145. SpringerVerlag, Berlin, 1987.

21. H. R. Malonek. Hypercomplex differentiability and its applications. In F. et al., editor, Clifford Algebras and Applications in Mathematical Physics, pages 141-150. Kluwer Academic Publishers, Netherlands, 1993.

22. V. P. Maslov. Operational Methods. "Nauka", Moscow, 1973. 
23. A. McIntosh and A. Pryde. A functional calculus for several commuting operators. Indiana Univ. Math. J., 36:421-439, 1987.

24. M. A. Rieffel. Quantization and $C^{*}$-algebras. In Doran [10], pages 68-97.

25. F. Riesz and B. Sz-Nagy. Functional Analysis. Ungar, New York, 1955.

26. I. Segal. $C^{*}$-algebras and quantization. In Doran [10], pages 55-65.

27. M. A. Shubin. Pseudodifferential Operators and Spectral Theory. Springer-Verlag, Berlin, 1987.

28. A. Sudbery. Quaternionic analysis. Math. Proc. Camb. Phil. Soc., 85:197-225, 1979.

29. J. L. Taylor. The analytic-functional calculus for several commuting operators. Acta Math., 125:1-38, 1970.

30. J. L. Taylor. A general framework for a multioperator functional calculus. Adv. Math., 9:183-252, 1972.

31. M. E. Taylor. Pseudodifferential Operators, volume 34 of Princeton Mathematical Series. Princeton University Press, Princeton, New Jersey, 1981.

32. M. E. Taylor. Noncommutative Harmonic Analysis, volume 22 of Math. Surv. and Monographs. American Mathematical Society, Providence, Rhode Island, 1986.

33. H. Weyl. The Theory of Groups and Quantum Mechanics. Dover, New York, 1950. 